\title{
Knowledge, attitude, and practices associated with the diagnosis and management of skin and soft-tissue infections among medical students, residents, and attending physicians
}

\author{
Norman Beatty, Jessica August, Joe Anthony Saenz, , David E. Nix², Kathryn R. Matthias², Mayar Al Mohajer³ \\ Departments of Medicine, ${ }^{1}$ Family and Community Medicine and ${ }^{2}$ Pharmacy Practice and Science, University of Arizona, Tucson, AZ, \\ ${ }^{3}$ Department of Medicine, Baylor College of Medicine, Houston, TX, USA
}

\begin{tabular}{|c|}
\hline Access this article online \\
\hline Website: www.avicennajmed.com \\
\hline DOI: 10.4103/ajm.AJM_200_17 \\
\hline Quick Response Code: \\
\hline \\
\hline
\end{tabular}

\begin{abstract}
Skin and soft-tissue infections (SSTIs) are commonly encountered by medical students, residents, and trainees. The Infectious Diseases Society of America (IDSA) has updated its recommendations regarding SSTI diagnosis and management in June 2014. We assessed knowledge, attitude, and practices toward diagnosis and management of SSTIs using an online survey. We disseminated the survey to medical students, residents, and attending physicians practicing in family and internal medicine department at a university-based hospital. A total of 103 surveys were completed out of 121 sent $(85.1 \%)$ between July 2015 and March 2016. There were nine medical questions in the survey. The mean of correct answers was $4.5 / 9 \pm 2.0$. Medical knowledge correlated with the level of education $(P<0.001)$ but not with subspecialty $(P=0.97)$. Around $35 \%$ were familiar with the updated IDSA guidelines pertaining to SSTIs. The majority $(85 \%)$ responded that the hospital staff would benefit from additional training and $75 \%$ agreed that more antibiotic stewardship education is needed. Our study shows that there are significant opportunities for development among students and physicians who encounter SSTIs.
\end{abstract}

Key words: Assessment, cellulitis, education, guidelines, survey

\section{INTRODUCTION}

It is estimated that skin and soft-tissue infections (SSTI) result in 14.2 million ambulatory visits (primary care, urgent care, and emergency department visits). ${ }^{[1]}$ Around 870,000 patients are admitted with SSTIs annually, with over half of them presenting with cellulitis or skin abscess. ${ }^{[2,3]}$ The majority of SSTIs are caused by Staphylococcus aureus or streptococci. ${ }^{[2]}$

Several studies have suggested high rates of inappropriate antibiotics for patients with SSTIs. In one study, $61 \%-80 \%$ of hospitalized patients with SSTI received unnecessary coverage against aerobic Gram-negative organisms and

Address for correspondence: Dr. Mayar Al Mohajer, Department of Medicine, Baylor College of Medicine,

1 Baylor Plaza, Houston, TX, USA.

E-mail: mohajer@bcm.edu
$73 \%-83 \%$ received unnecessary anaerobic coverage. In addition, most of the patients were treated with inappropriately prolonged therapy (antibiotic median duration of 13 days in patients with uncomplicated cellulitis or skin abscess). ${ }^{[3]}$ Another study showed that around half of the patients from ambulatory care settings with uncomplicated SSTI received inappropriate antibiotic therapy. ${ }^{[4]}$

This is an open access journal, and articles are distributed under the terms of the Creative Commons Attribution-NonCommercial-ShareAlike 4.0 License, which allows others to remix, tweak, and build upon the work non-commercially, as long as appropriate credit is given and the new creations are licensed under the identical terms.

For reprints contact: reprints@ @medknow.com

Cite this article as: Beatty N, August J, Saenz JA, Nix DE, Matthias KR, Mohajer MA. Knowledge, attitude, and practices associated with the diagnosis and management of skin and soft-tissue infections among medical students, residents, and attending physicians. Avicenna J Med 2018;8:104-6. 
In 2014, the Infectious Diseases Society of America (IDSA) updated its guidelines regarding the diagnosis and management of SSTIs. ${ }^{[5]}$ This update discussed various SSTI syndromes ranging from mild uncomplicated skin abscesses to necrotizing fasciitis. The guidelines also focused on physicians' clinical skills, their role in recognizing the possible causative pathogens, and prescribing appropriate antimicrobials.

\section{MATERIALS AND METHODS}

We performed a cross-sectional study using an electronic survey to assess the knowledge, attitude, and practices of medical students and physicians toward the diagnosis and management of SSTI. The study was approved by the Institutional Review Board.

The study was performed at an academic medical center from July 2015 till March 2016. We identified a total of 121 participants including medical students, residents, and attending physicians in internal medicine and family medicine department. Participants were invited by e-mail. We excluded members of the research team from participation in the study. There was no financial compensation for participants.

REDCap (Research Electronic Data Capture) software was used to create an online survey that included 35 close-ended questions. The knowledge portion of the survey assessed diagnosis and management of SSTIs including distinguishing purulent from nonpurulent cellulitis, outpatient versus inpatient management, recognizing necrotizing fasciitis, carbuncle, erysipelas, and lipodermatosclerosis (noninfection mimic), and appropriate therapies as per the SSTI IDSA guideline (based on syndrome, acuity, and suspected bacterial etiology). ${ }^{[5]}$ The attitude and practice component assessed reasons for choosing certain therapies over others, whether respondents were consulting clinical pharmacists to aid with management, opinions on antibiotic management and stewardship, and the need for further training.

The primary outcome of the study was the percentage of correct knowledge questions regarding the diagnosis and management of SSTIs. We hypothesized that knowledge scores would be higher for attending physicians compared with that of medical residents and students. Chi-squared test at $5 \%$ level of significance was used to compare subcategories (various levels of training) and internal medicine versus family medicine. STATA (StataCorp, College Station, Texas; version 13.1, USA) was used to perform statistical analysis.

\section{RESULTS}

A total of $103 / 121(85.1 \%)$ participants responded to the survey. They included 31 medical students, 57 medical residents, and 12 attending physicians. Three respondents chose not to state their level of training. A total of 28 family medicine and 43 internal medicine physicians (resident or attending) were surveyed.

\section{Medical knowledge}

There were nine medical questions in the survey. Results of medical knowledge questions are presented in Table 1 . The mean of correct answers was 4.5/9 \pm 2.0 . Medical knowledge correlated with the level of education $(P<0.001)$. Third-year medical residents and attending physicians had the highest means of correct answers (5.88/9 \pm 1.78 and 5.50/9 \pm 1.69 , respectively), while third-year medical students had the lowest mean of correct answers $(2.78 / 9 \pm 1.48)$. There was no relationship between medical knowledge and subspecialty $(P=0.97)$.

More than half of the participants (58.7\%) were unable to recognize the need for emergent surgery during a clinical presentation of necrotizing fasciitis. The majority of participants (53.8\%) felt that Staphylococci spp. rather than Streptococci spp. was the causative agent of nonpurulent cellulitis.

\section{Attilude and practices}

Most respondents (73\%) encountered between two and ten patients with a SSTI in the month prior to taking the survey. Cellulitis was the most common infection seen (95\%). Around three-quarters of the respondents (76.7\%) felt that most patients with SSTI can be managed as outpatients. The majority felt that hypotension or fever warranted admission to a hospital ( $97.8 \%$ and $65.2 \%$, respectively).

Fifty-eight participants (64.5\%) were confident with their abilities in diagnosing SSTI, while 42 (46.7\%) participants

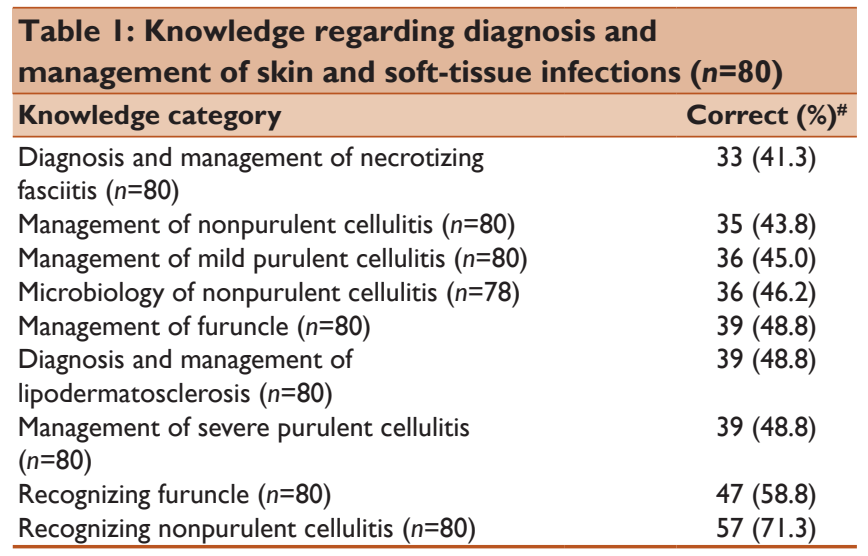


were confident in performing incision and drainage. Fifty-two participants (58.4\%) felt that they had sufficient training regarding SSTI management and only 31 (34.4\%) participants were familiar with the IDSA guidelines regarding the diagnosis and management of SSTIs. Familiarity with the IDSA guidelines was associated with the level of training $(P<0.001)$ but not with subspecialty $(P<0.39)$.

\section{Antibiotic stewardship}

Twenty respondents $(22.2 \%)$ felt that antibiotics were always necessary when treating patients with SSTIs. The most common antibiotics prescribed were oral trimethoprim/sulfamethoxazole $(56.8 \%)$, oral clindamycin (53.4\%), intravenous vancomycin (44.3\%), and oral cephalexin (43.2\%). Cost of antibiotics was considered as a factor in empiric coverage by 32 respondents (36\%).

The majority of participants (85.6\%) felt that the hospital would benefit from further teaching regarding antibiotic use in patients with SSTI and that clinical pharmacists and antibiotic stewardship team should be more involved in education efforts ( $60.7 \%$ and $74.7 \%$, respectively).

\section{DISCUSSION}

This study has demonstrated several knowledge gaps among students and physicians regarding the diagnosis and management of SSTIs. There should be greater focus in medical schools and residency programs on educating students and physicians on the updated IDSA guidelines regarding SSTIs, causative pathogens, clinical presentation, and how to treat cellulitis based on severity and causative agents. In addition, training should emphasize the need to recognize necrotizing fasciitis immediately and how to treat it appropriately given its life-threatening nature.

It is encouraging that participants have acknowledged their gaps in medical knowledge. They also recognized the need for further training and the role of consulting with clinical pharmacists and antibiotic stewardship education to aid in diagnosis and management. Based on these results, we are planning to design an online module aligned with the updated IDSA guidelines to educate our physicians with evidence-based recommendations regarding SSTIs.

Our study has several limitations. We assessed medical knowledge using nine simple clinical scenarios that might not reflect the real knowledge. In addition, we measured self-reported antibiotic prescription rather than the actual prescriptions. Our study was based on the responses of internal medicine and family medicine providers from only one academic medical center, which limits its generalizability to other hospitals.

\section{CONCLUSION}

There are significant opportunities for development among physicians who encounter SSTIs. Antibiotic stewardship, SSTI education for junior trainees, and promoting IDSA guidelines are areas needing improvement.

\section{Financial support and sponsorship}

Nil.

\section{Conflicts of interest}

There are no conflicts of interest.

\section{REFERENCES}

1. Hersh AL, Chambers HF, Maselli JH, Gonzales R. National trends in ambulatory visits and antibiotic prescribing for skin and soft-tissue infections. Arch Intern Med 2008;168:1585-91.

2. Edelsberg J, Taneja C, Zervos M, Haque N, Moore C, Reyes K, et al. Trends in US hospital admissions for skin and soft tissue infections. Emerg Infect Dis 2009;15:1516-8.

3. Jenkins TC, Sabel AL, Sarcone EE, Price CS, Mehler PS, Burman WJ, et al. Skin and soft-tissue infections requiring hospitalization at an academic medical center: Opportunities for antimicrobial stewardship. Clin Infect Dis 2010;51:895-903.

4. Hurley HJ, Knepper BC, Price CS, Mehler PS, Burman WJ, Jenkins TC, et al. Avoidable antibiotic exposure for uncomplicated skin and soft tissue infections in the ambulatory care setting. Am J Med 2013;126:1099-106.

5. Stevens DL, Bisno AL, Chambers HF, Dellinger EP, Goldstein EJ, Gorbach SL, et al. Practice guidelines for the diagnosis and management of skin and soft tissue infections: 2014 update by the Infectious Diseases Society of America. Clin Infect Dis 2014;59:147-59. 